\title{
Impugning conflict declarations
}

G reed corrupts. It perverts the masses and debauches that guardian of empiricism, objectivity and integrity—scientific research. Gratifyingly, in scientific publishing the balance is tipped in favor of truth by mandating openness and transparency through formal declarations of financial conflicts of interest. However, this precarious equilibrium was jolted by the release of a report entitled "Unrevealed: Non-disclosure of conflicts of interest in four leading medical and scientific journals" from the consumer watchdog Center for Science in the Public Interest (CSPI) on 12 July 2004. The popular press eagerly disseminated the report, which chastised four leading medical and scientific journals for not rigorously enforcing their conflict of interest disclosure policies and charged several authors with not disclosing financial support that the CSPI viewed as a conflict of interest. The seriousness of the issue suggests that the declaration is important. But how should this valuable piece of information be treated by scientific journals and their readers?

Science is objective, but scientists are not. In recognition of this reality, leading medical and scientific journals (including the Nature family of journals) now routinely require authors to declare conflicts of interest (mainly in terms of competing financial interest). This practice was instituted by journals in response to the increased percentage of research and researchers funded by sources that may financially benefit from particular research outcomes. Declaring all such associations introduces a level of transparency that allows readers to make an informed judgment on the significance of the reported findings. Such a dose of skepticism appears warranted, given reports that correlate positive conclusions with studies funded by industry (such as Als-Nielsen, B. et al. J. Am. Med. Assoc. 290, 921-928 (2003)). However, it is equally important that a study not be instinctively deemed flawed or biased simply because of a declaration of potential conflict or, conversely, be thought of more highly because of a lack of a financial conflict declaration. The latter studies could well be laced with personal, philosophical and circumstantial biases. These forms of bias are undeclared and are difficult to detect and control. In their own defense, scientists who conduct industry-funded research often claim that their studies are more closely scrutinized and are thus better conducted (see the associated response to the study mentioned above by Als-Nielson et al.). Thus, declaration of financial conflict of interest is but one useful piece of information to assess a report.

Should we despair, realizing that no scientific report is utterly objective and accurate? Of course not. Science will continue to advance as it always has-'intuitive' leaps followed by a series of laborious and confirmatory reiterations. Scientific readers are receptive to newly constructed propositions when the pillars on which they rest can be shown to be robust. Scientific journals serve as the forum for researchers to compare (sometimes surprising) new data with older more established results and to instigate independent tests. This built-in redundancy places less emphasis on any single piece of research with its inherent biases, and thus provides a mechanism of oversight and quality control.

The importance of being 'analytical and circumspect' is pertinent when assimilating the CSPI report. Although the purported aim of the CSPI - to promote accountability of authors in biomedical journals-is commendable, their study itself may be tainted. Reporting in the Globe and Mail (www.theglobeandmail.com) on 30 July 2004, Stephen Strauss revealed that one of the scientists identified by the CSPI study, Pierre Ayotte of the University of Laval, may have been unfairly incriminated for failing to disclose financial ties. The financial support that was reportedly undeclared came from the Canadian Chemical Producers Association, but it was provided to his host institute in a 'blind trust'. This donation constitutes $5 \%$ of the research institutes' revenue and the funding is distributed by an independent group of scientists. Ayotte did not know and, appropriately, could not declare such a contribution. If an author is not aware of financial support and thus is not influenced by it, then no conflict exists. In a paradoxical twist, the study in question reported an adverse effect of chemicallaced seafood consumed by mothers on the function of their babies' immune systems-not an outcome the chemical association could have relished. Thus, ironically, Ayotte is at ease with his conscience, whereas the CSPI report, and even its agenda, might be viewed with some skepticism.

Journals should rigorously ensure that declarations of competing financial interest are consistently obtained but should not resort to 'witch hunts'. Although some journals have declared that financial pursuit and scientific integrity are not compatible (Lancet 363, 2-3 (2004)), it is neither practical nor possible to stop this association. Leading scientists are likely to be enlisted by for-profit companies for their expertise. Thus, a more forward-looking approach is to provide reasonable guidelines for the declaration of conflicts, such that major opinion-makers may be heard and their views be debated under full disclosure. The guideline should be broad, such as that stipulated by the 2001 Nature editorial $(412,751)$ urging the declaration of "interests that could embarrass you were they to become publicly known after your work was published." Concurrently, the reprimands for noncompliance should be sufficiently severe to serve as a legitimate deterrent. In this case, public highlighting of nondisclosure may be considered. Equally important, however, the reader of scientific reports must put declarations in perspective. Although greed may be discouraged by a conflict of interest pronouncement, a probing mind is needed to ultimately find the truth. The French Nobel laureate André Gide reminded us to "believe those that are seeking the truth; doubt those who find it"- a probing declaration, indeed. 